$R M x A C, \mathbf{5 3}, 137-139(2021)$

(c) 2021: Instituto de Astronomía, Universidad Nacional Autónoma de México

https://doi.org/10.22201/ia.14052059p.2021.53.27

\title{
TAOS II: THE ROBOTIC OPERATIONS
}

Joel H. Castro-Chacón ${ }^{1}$, Matthew Lehner ${ }^{2,4,5}$, Mauricio Reyes-Ruiz ${ }^{3}$, Benjamín Hernández ${ }^{3}$, Charles Alcock ${ }^{6}$, Chung-Kai Huang ${ }^{2,7}$, Kem H. Cook ${ }^{2}$, John C. Geary ${ }^{5}$, L. Figueroa ${ }^{3}$, C. Guerrero ${ }^{3}$, J. B. Hernández-Águila ${ }^{3}$, Juan C. Narvaez ${ }^{3}$, Timothy Norton ${ }^{5}$, Fernando Quiroz ${ }^{3}$, E. Sánchez ${ }^{3}$, Shiang-Yu Wang ${ }^{2}$, José S. Silva ${ }^{1}$, Andrew Szentgyorgyi ${ }^{5}$, You-Hua $\mathrm{Chu}^{2}$, Wei-Ling $\mathrm{Yen}^{2}$, Wen-Ping $\mathrm{Chen}^{7}$, and Zhi-Wei Zhang ${ }^{2}$

\section{RESUMEN}

En este trabajo, presentamos el procedimeinto general para llevar a cabo las observaciones robóticas del Censo Automatizado de Ocultaciones para objetos Transneptunianos (TAOS II por sus siglas en inglés). El objetivo del proyecto es detectar objetos transneptunianos (TNOs) pequeños por medio de ocultaciones estelares fortuitas. Para esto, TAOS II operará con tres telescopios de $1.3 \mathrm{~m}$ equipados con cámaras CMOS capaces de leer 10,000 estrellas en múltiples subaperturas a $20 \mathrm{~Hz}$. A tal cadencia, será posible identificar las características de difracción en las curvas de luz, lo cual ayudará a estimar la distancia y tamaño del objeto en cada evento de ocultación. TAOS II está instalado en el Observatorio Astronómico Nacional de San Pedro Mártir en Ensenada, México (OAN-SPM). El sitio posee muy buenas condiciones para observación, típicamente alrededor de 260 noches útilies por año. Aquí describimos los diferentes procesos que deben llevarse a cabo en una noche de observación: encendido y apagado del sistema, monitoreo de las condiciones del cielo, adquisición de imágenes de calibración, selección de campos, apuntado simultáneo, sicronización de las cámaras, determinación de tamaños de apertura y posciones, y adquisición de imágenes a alta velocidad.

\section{ABSTRACT}

In this work, we present the general procedure for the robotic observations of the Transneptunian Automated Occultation Survey (TAOS II). The project aims to detect small TNOs (Transneptunian Objects) by serendipitous stellar occultations. To do so TAOS II will operate three $1.3 \mathrm{~m}$ telescopes equipped with CMOS cameras which are able to read about 10,000 stars in multiple sub-apertures at a $20 \mathrm{~Hz}$ cadence. At such rates, it will be possible to identify diffraction features in the lightcurves, helping us to estimate a distance and object size to each occultation event. TAOS II is installed in the Observatorio Astronómico Nacional in San Pedro Mártir, Ensenada, México (OAN-SPM). The site has good observing conditions, typically with around 260 useful nights per year. Here, we describe the different process to be performed in a typical observing night: system start up and shut down, monitoring observing conditions, acquisition of calibration images, field selection, pointing, camera synchronization, determination of aperture sizes and positions, and high speed image acquisition.

Key Words: Kuiper belt: general — occultations - surveys - telescopes

\footnotetext{
${ }^{1}$ CONACYT - Instituto de Astronomía, Universidad Nacional Autónoma de México. Km. 107 Carretera TijuanaEnsenada, Ensenada Baja California, México. C.P. 22860 (joelhcch@astro.unam.mx).

${ }^{2}$ Academia Sinica Institute of Astronomy and Astrophysics, $11 \mathrm{~F}$ of AS/NTU Astronomy-Mathematics Building, No.1, Sec. 4, Roosevelt Road, Taipei 10617, Taiwan, R.O.C.

${ }^{3}$ Instituto de Astronomía, Universidad Nacional Autónoma de México, Km. 107 Carretera Tijuana-Ensenada, Ensenada Baja California, México. C.P. 22860.

${ }^{4}$ Department of Physics and Astronomy, University of Pennsylvania, 209 South 33rd Street, Philadelphia, PA 19125, USA.

${ }^{5}$ Harvard-Smithsonian Center for Astrophysics, 60 Garden Street, Cambridge, MA 02138, USA.

${ }^{6}$ Herzberg Astronomy and Astrophysics Research Centre, National Research Council of Canada, 5071 West Saanich Road, Victoria, British Columbia V9E 2E7, Canada.

${ }^{7}$ Institute of Astronomy, National Central University, 300 Jhongda Road, Jhongli 32054, Taiwan, R.O.C.

${ }^{8}$ Department of Physics and Astronomy, University of Victoria, Building, 3800 Finnerty Road, Victoria, BC V8P 5C2, Canada.
}

\section{INTRODUCTION}

TAOS II is the product of collaboration between institutions in Taiwan (ASIAA), Mexico (UNAM), the United States (CfASAO) and Canada (NRC). The project will operate 3 robotic $1.3 \mathrm{~m}$ wide field telescopes in the OAN-SPM. Each telescope will have a scientific CMOS based, $80 \mathrm{Mpx}$ camera capable of taking images at $20 \mathrm{~Hz}$ from more than 10,000 stars simultaneously over a 2.3 square degree field (Lehner et al., 2018). The main objective of TAOS II is to provide observational constraints to the size distribution of TNOs for improving the models of the Solar System evolution (Benavidez \& Campo, 2009; Kenyon \& Bromley, 2009) since it is an open question and more measurements are needed (Arimatsu et al., 2019). The survey relies in robotic 


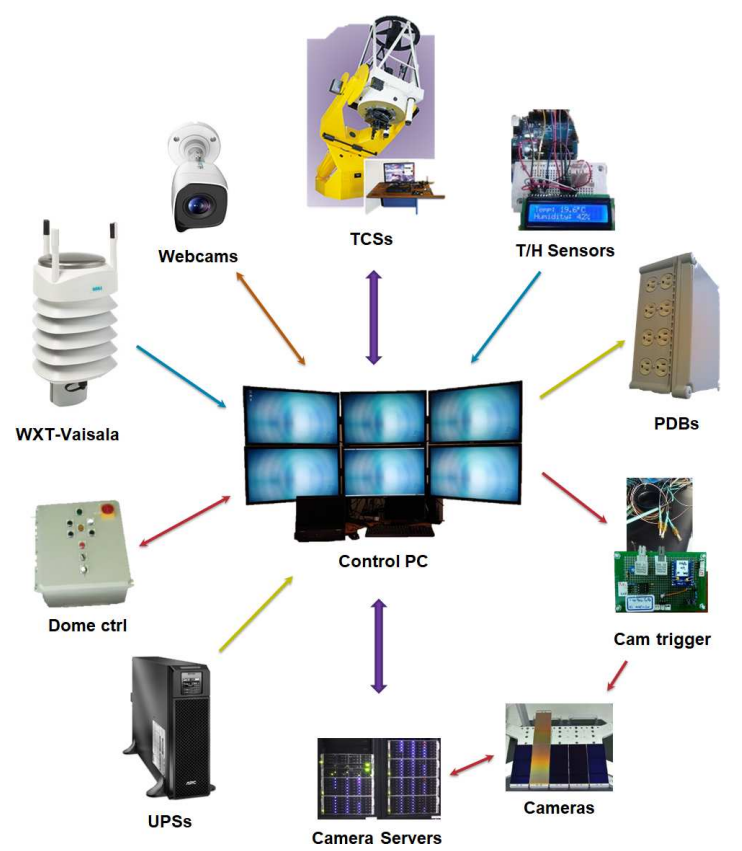

Fig. 1. Set of main instruments for the control of the robotic observation. The control PC will be connected to different peripherals in order to: 1)receive the status from the dome, UPSs, weather stations, temperature and humidity sensors; 2) send signals to the PDBs (Power Distribution Boxes) and the camera triggers; 3) keeping two-way communication with the TCSs, the camera servers, the webcams and the dome control. The whole set of peripherals is installed on each site.

observations to collect all data, the human intervention, in sites, is aimed to be reduced to maintenance, so all details must be determined in advance and prevented to assure the success of the observing plan. The data will be processed with a fast-photometry pipeline to produce basic lightcurves, as a first approach to obtain occultation signals and generate alerts for following up. Raw data and lightcurves will be copied from the OAN-SPM to a data center in Ensenada, once this process is completed, a checksum process will determine when it is safe to erase the OAN-SPM data. The data from Ensenada will be sent to a storage mirror in Canada, so we will keep all data in both places. With all images in the data center we will perform a more reliable photometry reduction (Sánchez et al., 2019) in order to be able of investigating the occultation features and other signals for side science.

\section{INSTRUMENTATION}

In order to control all the subsystems involved in the observation is necessary to know the status of each instrument.

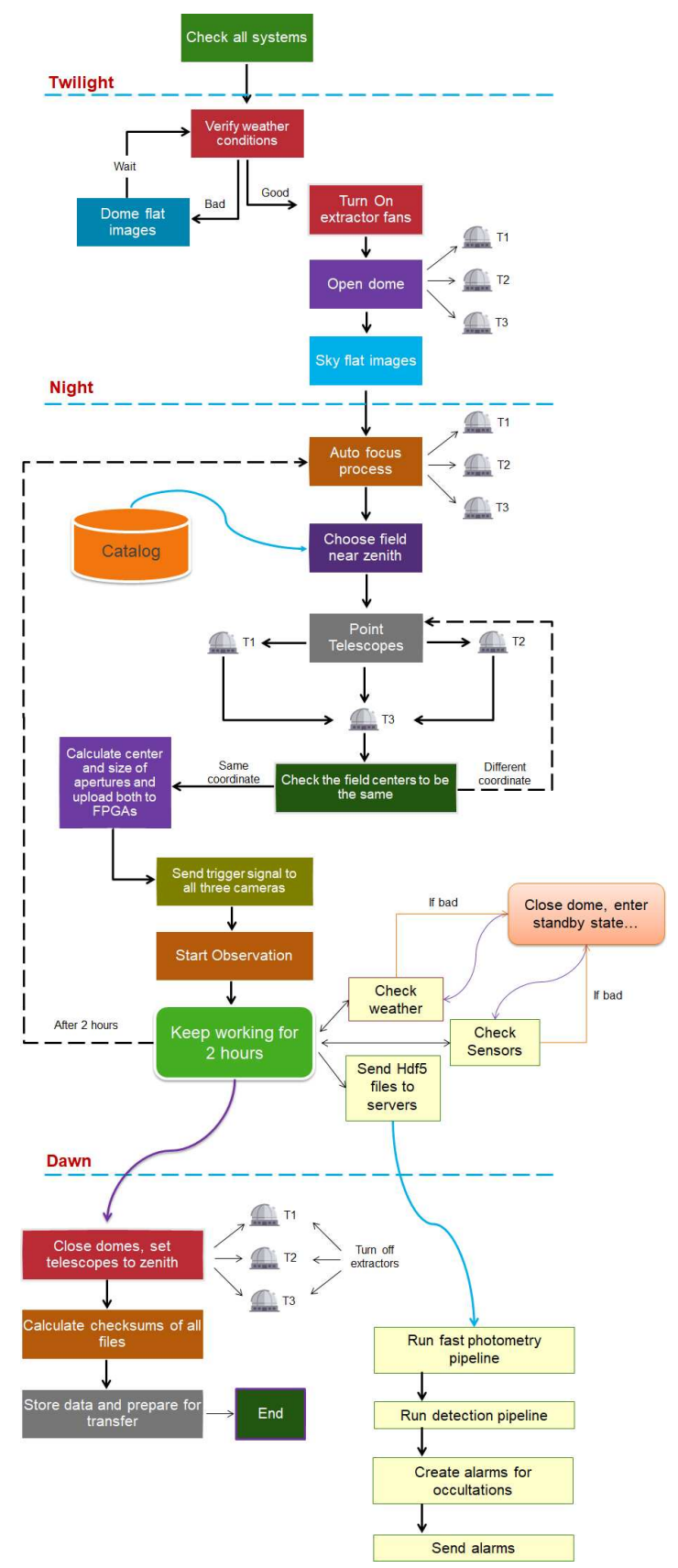

Fig. 2. Flowchart depicting the robotic operations. Before the operation starts all systems showed in Fig. 1 have to be checked, if some of them fails the system will shut down. The calibration images will be acquired at twilight, then the observation will take place up to 4 or 5 fields per night and at dawn the whole system will be set to standby.

In Fig. 1 all subsystems and their connections are depicted. TAOS II will allow to monitor all signals by using a central computer with six monitors, this $\mathrm{PC}$ will be running from the control room at the 


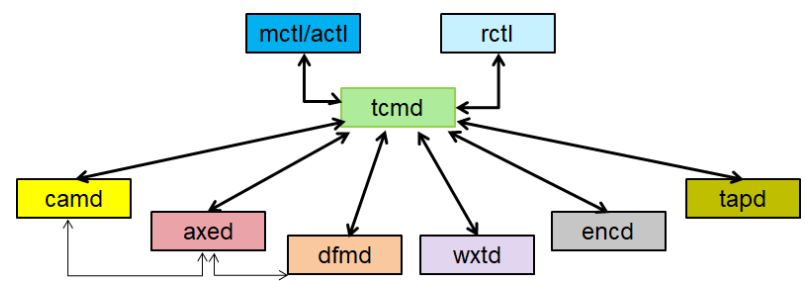

Fig. 3. Chain of command for daemons from the control software. The main daemon is the tcmd and will run in the control PC, while the others will run as instances for each site. The arrows indicate always a two way communication with tcmd and among other daemons.

OAN-SPM, but a shared shell will be available to replicate the same information in Taipei and Ensenada.

\section{OPERATION FLOWCHART}

In Fig. 2 we show the flowchart in an ordinary observation night. Each sensor will be constantly measured by the system, so it will decide when to open and close the telescopes. The operations will start on twilight by obtaining sky-flats and/or domeflats. Then, the autofocus process will be performed in all telescopes. Once it is finished, the telescopes will point to the closest field, form the catalog, to zenith, so the system will check that all the stars to be observed are visible in all three detectors. Then the list of regions will be generated and the image acquisition will run in that field per two hours.

\section{CONTROL SOFTWARE}

The control software relies in a group of "daemons" that will be communicating with a main one called tcmd. In Fig. 3 a scheme of communication among all "daemons" is depicted, here we list all and their function.

- tcmd: main control daemon. Interface between shell and hardware control daemons.

- camd: camera image acquisition control.

- $d f m d$ : interface to DFM TCS (dome and telescope control).

- axed: interface between camera data and telescope.

- control: guiding, autofocus, pointing model acquisition and analysis.

- wxtd: weather station control.

- encd: ion pump, temperature controller, vacuum gauge, FPGA power supply, various temperature sensors (ambient, primary and secondary mirrors, etc.), extractor fans, and dome and telescope power supplies.

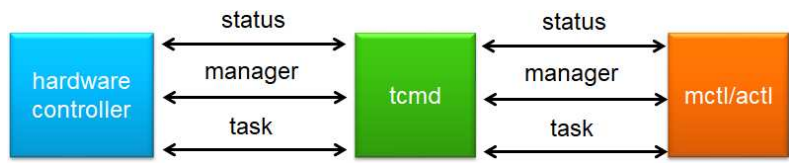

Fig. 4. Communication among principal daemons. Three types of messages are allowed among daemons: status, management and regular tasks. tcmd will control manually or automatically all the hardware systems.

- $m c t l / a c t l$ : bash shell with loadable extensions to manually control the various hardware components.

- rctl: robotic control of system operations, including observation scheduling, response to power outages and bad weather events, and data analysis pipeline management.

- tapd: TAOS II data analysis pipeline manager.

In order to assure proper communication, three connections to each daemon in asynchronous operation will have separate threads for each connection, see fig 4 . Here we list the functions of each connection.

- status: get daemon status info (telescope position, camera temperature, etc.)

- manager: "out of band" commands (shutdown, abort, ping, etc.)

- task: execute a command (open dome, move telescope, take image, etc.)

\section{CONCLUSIONS}

We have presented the instrumental elements, the flowchart for a single night of robotic operations, and a general scheme of the control software that will allow to carry out the robotic operations of TAOS II.

For fulfilling its scientific goals, TAOS II requires a complete failure proof control system, since we expect not to depend on human intervention

TAOS II is a unique system, not only due to the implementation of fast photometry by observing multiple sub-apertures simultaneously, but because there are three telescopes that must be completely synchronized.

Acknowledgments: Joel Castro acknowledges the support of CONACYT through the projects: Cátedras (219) and CB 283800.

\section{REFERENCES}

Arimatsu, K., Tsumura, K., Usui, F., et al. 2019, NatAs, 3, 301

Benavidez, P. G. \& Campo Bagatin, A. 2009, P\&SS, 57, 201

Kenyon, S. J. \& Bromley, B. C. 2009, ApJ, 690, 140

Lehner, M. J., Wang, S.-Y., Reyes-Ruíz, M., et al. 2018, SPIE, 10700, 4

Sánchez, E., Castro-Chacón, J. H., Silva, J. S., et al. 2019, $\mathrm{A} \& \mathrm{C}, 26,1$ 